\title{
Allium Jesdianum Extract Improve Acetaminophen- Induced Hepatic Failure through Inhibition of Oxidative/Nitrosative Stress
}

\author{
Zohreh Sohrabinezhad 1,2, Dara Dastan 1,3, Sara Soleimani Asl 4, Amir Nili-Ah- \\ madabadi ${ }^{1,2 *}$
}

${ }^{1}$ Medicinal Plants and Natural Products Research Center, Hamadan University of Medical Sciences, Hamadan, Iran

2 Department of Pharmacology and Toxicology, School of Pharmacy, Hamadan University of Medical Sciences, Hamadan, Iran

${ }^{3}$ Department of Pharmacognosy, School of Pharmacy, Hamadan University of Medical Sciences, Hamadan, Iran

${ }^{4}$ Anatomy Department, School of Medicine, Hamadan University of Medical Sciences, Hamadan, Iran

\section{Key Words}

Allium jesdianum, Oxidative stress, Acetaminophen, Hepatotoxicity

\begin{abstract}
Objectives: Allium jesdianum $(\mathrm{Aj})$ is a medicinal plant that has highlighted pharmacological features. In this study, the effects of Aj extract were examined on acetaminophen (APAP)-induced hepatic failure in rats.
\end{abstract}

Methods: Methanolic fraction of hydro-alcoholic extract of Aj was obtained by silica gel column chromatography method. Animals were randomly divided into four groups each containing six rats and treated by gavage as follows: the first and second groups received normal saline, the third and fourth groups were received with 50 and $100 \mathrm{mg} / \mathrm{kg}$ of Aj extract, respectively. After two consecutive weeks, the groups 2-4 were given a single dose of APAP $(2 \mathrm{~g} / \mathrm{kg})$. After 48 hours, blood and liver samples were collected for biochemical and histological examinations.

Results: The findings of the study demonstrated that APAP caused a significant increase in ALT $(\mathrm{P}<0.001)$, AST $(\mathrm{P}<0.001), \operatorname{LDH}(\mathrm{P}<0.001)$, ALP $(\mathrm{P}<0.001)$ serum

Received: Mar 01, 2019 Reviewed: Jul 18, $2019 \quad$ Accepted: Nov 25, 2019

(C) This is an Open-Access article distributed under the terms of the Creative Common Attribution Non-Commercial License (http://creativecommons.org/licenses/by-nc/4.0/) which permits unrestricted noncommercial use, distribution, and reproduction in any medium, provided the original work is properly cited.

(2) This paper meets the requirements of KS X ISO 9706, ISO 9706-1994 and ANSI/NISO Z39.48-1992 (Permanence of Paper). levels, hepatic lipid peroxidation (LPO; $\mathrm{P}<0.001)$ and nitric oxide (NO; $\mathrm{P}<0.001)$. In this regard, APAP led to the depletion of the total antioxidant capacity (TAC; P $<0.001$ ), glutathione and total thiol groups (TTGs; $\mathrm{P}<$ 0.001 ), and structural change in the liver. In the $\mathrm{Aj}$ extract groups, a considerable improvement was found in the hepatic function alongside the histopathologic changes.

Conclusion: This investigation indicated that the influential effects of Aj extract in APAP-induced hepatic failure might depend on its effect on improving oxidant/antioxidant balance in hepatic tissue.

\section{Introduction}

Acetaminophen (acetyl-para-aminophenol; APAP), has been used extensively but it can cause hepatotoxicity when more than therapeutic doses are used [1]. About $80 \%$ of APAP is conjugated directly to sulfated and glucuronidated metabolites and secreted in bile or urine. A slight portion of APAP (about 5\%-10\%) is metabolized to $\mathrm{N}$-acetyl-para-benzo-quinone imine (NAPQI) by hepatic cytochrome CYP 2E1 which is an extremely reactive toxic metabolite $[1,2]$. This electrophilic metabolite can binds covalently to some of the intracellular molecules such as DNA, deplete glutathione (GSH) which may cause oxidative stress, and alter calcium and/or thiol status in hepatocellular, all of which cause hepatic failure $[2,3]$.

"Corresponding Author

Amir Nili-Ahmadabadi. Department of Pharmacology and Toxicology, School of Pharmacy, Hamadan University of Medical Sciences, Hamadan, Iran. P.O. Box 8678-3-65178, Tel: +98-813-838-0031 Fax: +98-813-838-0031

Email: amirnili54@gmail.com 
Medicinal herbs and/or herbal supplements have been mostly applied to sustain or upgrade health, and the community believes that natural herbal compounds have no side effects and they are safe. The World Health Organization (WHO) recited that $80 \%$ of people in developing countries use herbal medicine to treat their health problems [4-6]. Among these, many studies have encouraged the use of Allium species as food and/or medicine so that people all over the world consume Allium vegetables especially onions and garlic, as a part of their daily diet. Various studies have been conducted to investigate the potential health-promoting effects of Allium species [79]. Beneficial properties of these species on hypertension, cardiovascular risk factors and lipid profile seemed to be based on various recent meta-analyses [10-13]. Furthermore, several studies have shown that some of the Allium species contain antioxidant properties and can inhibit oxidative damage induced by oxidants agents [14, 15].

Allium jesdianum (Aj) is a type of the Allium that can be found in the Zagros Mountains of Iran. Recently, some useful effects of $\mathrm{Aj}$ like antibacterial and antifungal activity have been confirmed $[16,17]$. However, there has not been a lot of information about the therapeutic effects of $\mathrm{Aj}$ on hepatic function. Hence, the aim of the present study was to examine the effects of $\mathrm{Aj}$ extract against APAP-induced liver dysfunction in male rats.

\section{Materials and methods}

\subsection{Chemicals}

Acetaminophen (98\% purity, CAS\#: 103-90-2), 5,5'-Dithiobis (2-nitrobenzoic acid) (DTNB), N-(1-naphthyl) ethylenediamine dihydrochloride (NED), bovine serum albumin (BSA), 2-thiobarbituric acid (TBA), and 2,4,6-Tris (2-pyridyl)-s-triazine (TPTZ) were purchased from Sigma-Aldrich Chemical Company (St. Louis, MO, USA).

\subsection{Plant materials: collection and extrac- tion procedure}

The Aj was gathered from the Zagros Mountains, kurdistan province, Iran. The collected plant was identified by herbarium unit, faculty of pharmacy, Hamadan University of Medical Sciences (HUMS), Hamadan, Iran with the code number of (NO: 403). The plant samples were dried and powdered. The extraction process was conducted using methanol and water (1:1) for 72 hours. Then, the resulting extract was filtered; and condensed with a rotary evaporator. The total extraction process was repeated three times. In the next stage, n-hexane, chloroform, ethyl acetate and methanol fractions of hydroalcoholic extract were prepared, respectively by column chromatography. For this purpose, a column with dimensions of $8 \times 17 \mathrm{~cm}$ was used with a silica gel (70-30 mesh) as a solid phase. Finally, the Ferric reducing/antioxidant power assay (FRAP) was performed on the fractions and the methanolic fraction as an effective fraction was selected and maintained at the temperature of $4^{\circ} \mathrm{C}$.

\subsection{Animals}

Male Wistar rats $(250 \pm 20 \mathrm{~g})$ were taken from the animal house of HUMS, Hamadan, Iran, and quarantined for one week before use. The animals were monitored in the suitable laboratory condition that had the cycle of the 12 $\mathrm{h}$ light/dark at the temperature of $22-25^{\circ} \mathrm{C}$, and were provided with the standard diet and water ad libitum. The investigation procedure was confirmed by the Ethics Committee of HUMS (ethical ID: IR.UMSHA.REC.1396.336).

\subsection{Safety dose determination}

According to pilot studies, the safety dose of $\mathrm{Aj}$ extract was evaluated to be in the range of $50-200 \mathrm{mg} / \mathrm{kg}$ through determining hepatic enzymes activity.

\subsection{Experimental protocol and groups}

The animals were divided into four groups of six, as follows: the first and second groups received normal saline, the third and fourth groups were treated with 50 and 100 $\mathrm{mg} / \mathrm{kg}$ of $\mathrm{Aj}$ extract, respectively. After two weeks, the groups 2-4 were given a single dose of APAP ( $2 \mathrm{~g} / \mathrm{kg})$. After 48 hours, the rats were anaesthetized with ketamine and xylazine (5:1 ratio) [18]. Following laparotomy surgery, blood specimens were obtained from the animals' heart, allowed to clot, and for $10 \mathrm{~min}$, they were centrifuged at $3000 \mathrm{~g}$ to acquire clear serum. The samples of the serum were kept at $-20^{\circ} \mathrm{C}$ for biochemical examinations. The liver was isolated from rats, and part of the hepatic tissue was homogenized in phosphate-buffered saline (PBS, pH 7.4). After centrifuging samples at $3000 \mathrm{~g} / 4^{\circ} \mathrm{C}$ for $10 \mathrm{~min}$, its supernatant was removed for biochemical examinations. Another part of the hepatic tissue was fixed in formalin (10\%) for histopathological examination.

\subsection{Serum enzymes analysis}

Enzymatic colorimetric kits were used to assay serum alanine transaminase (ALT), aspartate transaminase (AST), lactate dehydrogenase (LDH) and alkaline phosphatase (ALP) activity (Pars Azmun kit, Iran).

\subsection{Nitric oxide assay}

Griess reagent ( $1 \%$ sulfanilamide, $0.1 \%$ NED, and $2.5 \%$ phosphoric acid) was used to identify liver nitric oxide (NO) as described by Nili-Ahmadabadi et al. [19]. 


\subsection{Lipid peroxidation assay}

Thiobarbituric acid-reactive substances (TBARS) method was used for lipid peroxidation (LPO) assay, as described by Nili-Ahmadabadi et al. [19]. Briefly, homogenized liver tissue were mixed with TBA $(0.2 \%)$ in $\mathrm{H} 2 \mathrm{SO} 4$ $(0.05 \mathrm{M})$ and heated for $30 \mathrm{~min}$ in boiling water bath. Byproducts of LPO were extracted by n-butanol and absorbance was determined at $532 \mathrm{~nm}$. Malondialdehyde (MDA) was used as a standard and data were expressed as nmol/ mg protein.

\subsection{Total antioxidant capacity assay}

Total antioxidant capacity (TAC) was determined in hepatic tissue by measuring their ability to reduce $\mathrm{Fe}^{+}$to $\mathrm{Fe}^{+}$. The reaction between TPTZ reagent and $\mathrm{Fe} 2^{+}$gives a blue color with a maximum absorbance at $593 \mathrm{~nm}$ [20]. Data were expressed as $\mathrm{nmol} / \mathrm{mg}$ protein.

\subsection{Total thiol groups assay}

Total thiol groups (TTGs) were determined spectrophotometrically by DTNB reagent. This reagent reacts with the thiol molecules to yield a yellow colored compound, which has a peak absorbance at $412 \mathrm{~nm}$ [21]. Data were expressed as $\mu \mathrm{mol} / \mathrm{mg}$ protein.

\subsection{Glutathione assay}

The GSH hepatic levels were measured according to the kit brochure from ZellBio GmbH Company, Germany. Data were expressed as $\mathrm{nmol} / \mathrm{mg}$ protein.

\subsection{Protein assay}

At the end of each experiment, protein content was as- sayed using the Bradford method in the crude homogenate of hepatic tissue. The BSA was used as standard to calculate protein content [20].

\subsection{Histopathological examination}

A part of the hepatic tissue was removed from the each rat and held in $10 \%$ formalin for at least $24 \mathrm{~h}$. The paraffin-embedded block was obtained by automatic tissue processor, Autotechnique. In the next stage, the tissue was cut into $4 \mu \mathrm{m}$ thick parts by a rotating microtome with at least three cross-sections of tissue on each slide. The samples were stained by hematoxylin and eosin (H\&E) dye for histopathological analysis [22].

\subsection{Statistical analysis}

The data were described using the mean and standard deviation (SD). The differences were determined, at 95\% confidence, using the analysis of variance (ANOVA) and Turkey's test to account for multiple comparisons. All analyses were executed by Graph Pad Prism software (version 6.0).

\section{Results}

\subsection{The efficiency of $A j$ fractions}

The yield of hydroalcoholic extract was $32.67 \%$, and the yield of n-hexane, chloroform, ethyl acetate and the methanolic fraction of hydroalcoholic extracts were $0.94,0.39$, 0.79 and $74.78 \%$, respectively.

\subsection{The effects of $A j$ extract on liver func- tion biomarkers}

The results indicated no sign of toxicity up to $100 \mathrm{mg} / \mathrm{kg}$ of $\mathrm{Aj}$ extract. There was an increase in ALP $(\mathrm{P}<0.001)$ and $\mathrm{LDH}(\mathrm{P}<0.001)$ serum activity in the dose of $200 \mathrm{mg} / \mathrm{kg} \mathrm{Aj}$

Table 1 The effects of Allium jesdianum (Aj) extract on liver function tests.

\begin{tabular}{|c|c|c|c|c|}
\hline Groups & $\begin{array}{c}\text { ALT activity } \\
\text { (U/L) }\end{array}$ & $\begin{array}{c}\text { AST activity } \\
\text { (U/L) }\end{array}$ & $\begin{array}{c}\text { ALP activity } \\
\text { (U/L) }\end{array}$ & $\begin{array}{c}\text { LDH activity } \\
\text { (U/L) }\end{array}$ \\
\hline Control & $43.38 \pm 7.04$ & $74.70 \pm 11.50$ & $120.66 \pm 12.48$ & $157.00 \pm 10.30$ \\
\hline$A j$ extract $(50 \mathrm{mg} / \mathrm{kg})$ & $37.89 \pm 5.78$ & $70.08 \pm 10.7$ & $128.57 \pm 5.68$ & $155.99 \pm 10.45$ \\
\hline$A j$ extract $(100 \mathrm{mg} / \mathrm{kg})$ & $40.53 \pm 3.79$ & $74.98 \pm 7.78$ & $124.04 \pm 8.13$ & $163.98 \pm 10.47$ \\
\hline$A j$ extract $(200 \mathrm{mg} / \mathrm{kg})$ & $43.05 \pm 6.83$ & $79.20 \pm 10.71$ & $138.33 \pm 7.07^{* * *}$ & $259.04 \pm 18.37^{* * *}$ \\
\hline
\end{tabular}


extract in comparison with control (Table 1). The administration of APAP significantly increased ALT $(\mathrm{P}<0.001)$, AST $(\mathrm{P}<0.001)$, ALP $(\mathrm{P}<0.001)$ and LDH $(\mathrm{P}<0.001)$ serum activity in comparison with the control group (Fig. 1A-1D, respectively). The serum levels of liver enzymes (ALT, AST, LDH and ALP) in animals exposed to APAP could be decreased by the Aj extract.

\subsection{The effects of $\boldsymbol{A j}$ extract on oxidative stress biomarkers}

Following APAP administration, the levels of LPO (Fig. $2 \mathrm{~A})$ and NO (Fig. 2B) were increased $(\mathrm{P}<0.001)$ and TAC (Fig. 3A), as well as TTGs and GSH (Fig. 3B and 3C, respec- tively) levels, were decreased in liver tissue compared to control group $(\mathrm{P}<0.001)$. In addition, a significant improvement was observed in TTGs $(\mathrm{P}<0.01)$, and GSH $(\mathrm{P}$ $<0.05)$ levels following pre-treatment with the Aj extract (Fig. 3B and 3C, respectively). In this regard, the levels of NO and LPO were decreased in treatment groups $(\mathrm{P}<$ 0.001).

\subsection{The effects of $A j$ extract on oxidative stress biomarkers}

Following APAP administration, the levels of LPO (Fig. $2 \mathrm{~A})$ and NO (Fig. 2B) were increased $(\mathrm{P}<0.001)$ and TAC (Fig. 3A), as well as TTGs and GSH (Fig. 3B and 3C, respec-
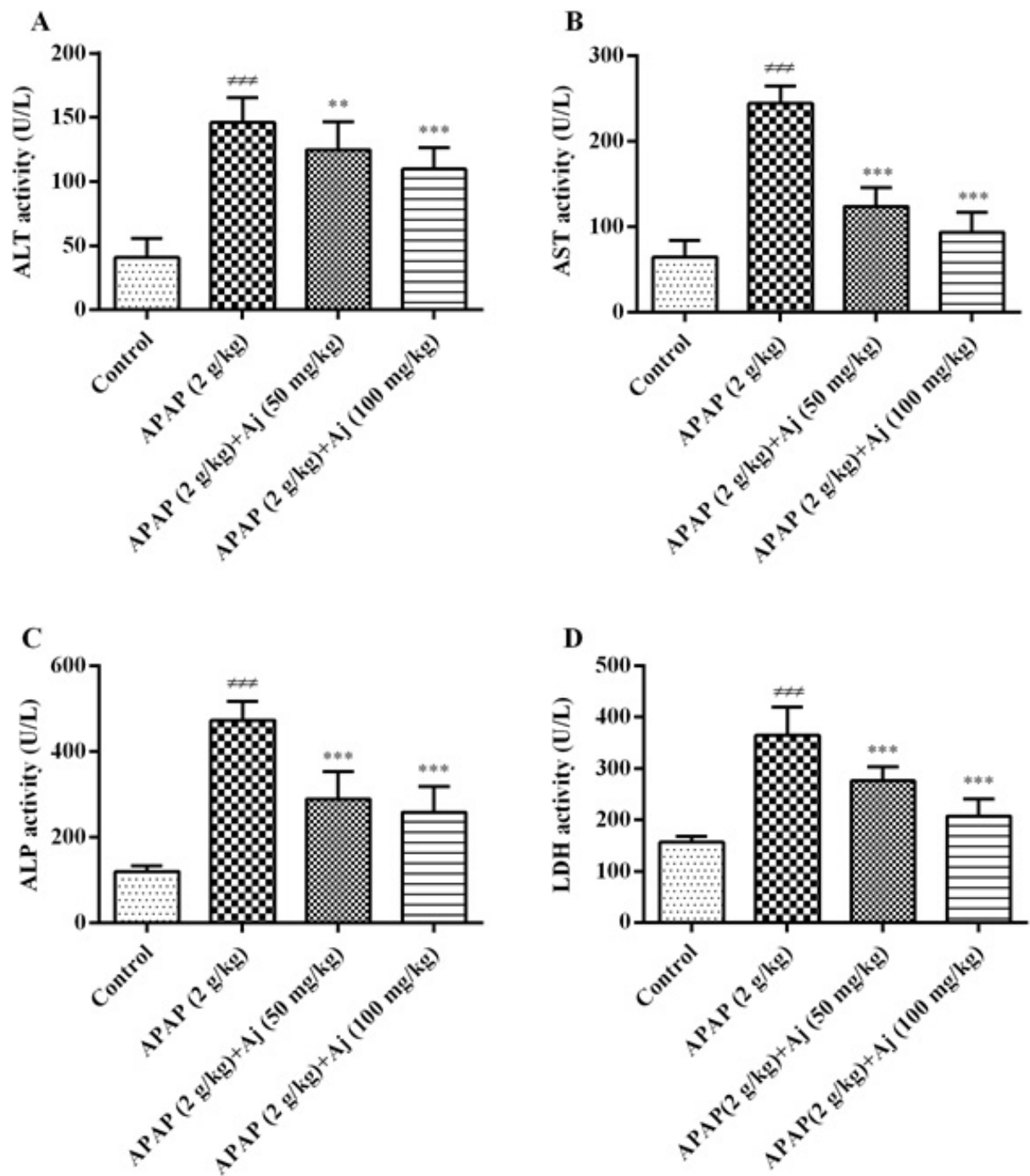

Figure 1 Effects of Allium Jesdianum ( $A j$ ) on serum enzymes of acetaminophen (APAP)-exposed wistar rat. Statistical analysis used one-way ANOVA with Tukey's test. Values are expressed as means $\pm \mathrm{SD}, \mathrm{n}=6$ for each group. $\neq \neq \neq P<0.001$ vs control group; ** $P<0.01$ and ${ }^{* * *} P<0.001$ vs APAP group. ALT: alanine aminotransferase (A); AST: aspartate aminotransferase (B); ALP: alkaline phosphatase (C); LDH: lactate dehydrogenase (D). 

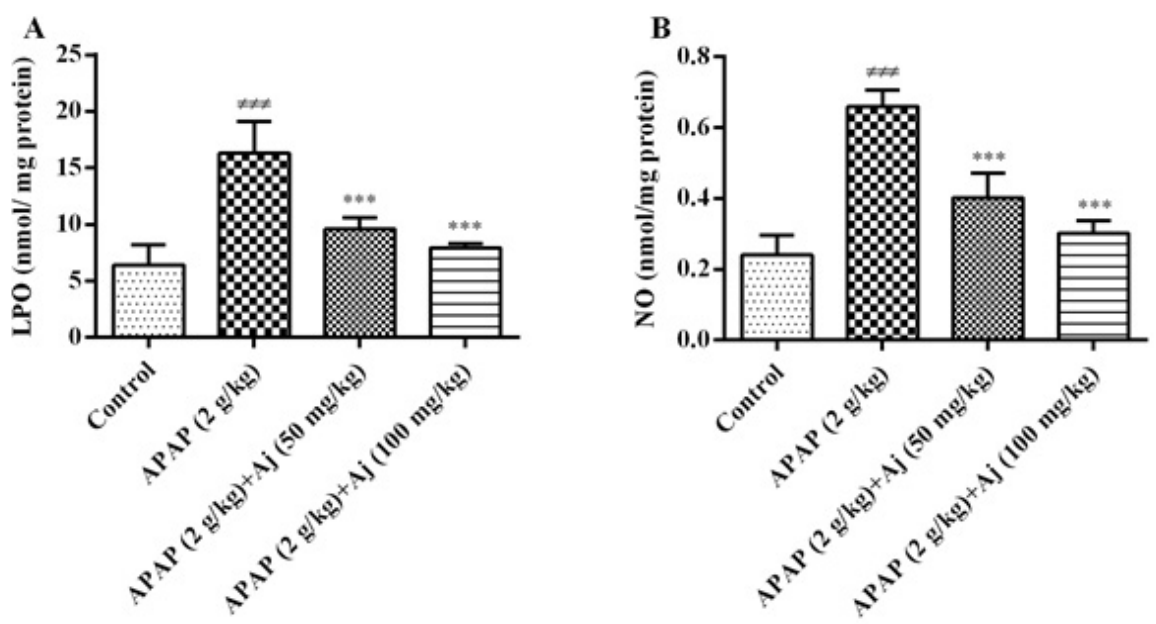

Figure 2 Effects of Allium jesdianum ( $A j$ ) on oxidant biomarkers of acetaminophen (APAP)-exposed wistar rat. Statistical analysis used one-way ANOVA with Tukey's test. Values are expressed as means $\pm \mathrm{SD}, \mathrm{n}=6$ for each group. $\neq \neq \neq P<0.001$ vs control group; *** $P<0.01$ vs APAP group. LPO: lipid peroxidation (A); NO: nitric oxide (B).
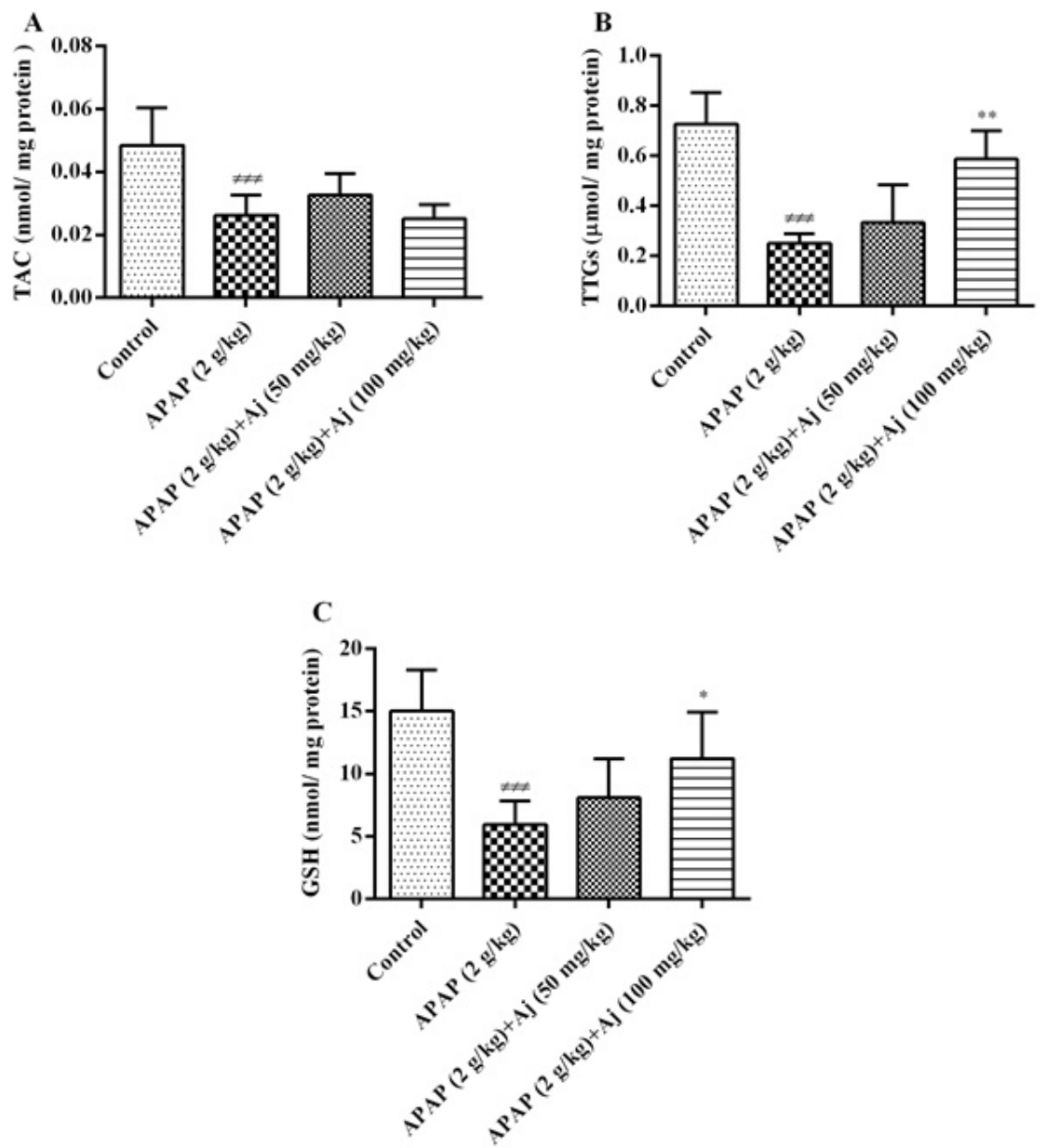

Figure 3 Effects of Allium jesdianum ( $A j$ ) on antioxidative biomarkers of acetaminophen (APAP)-exposed wistar rat. Statistical analysis used one-way ANOVA with Tukey's test. Values are expressed as means $\pm \mathrm{SD}, \mathrm{n}=6$ for each group. $\neq \neq \neq \mathrm{P}<0.001$ vs control group; ${ }^{*} P<0.05$, ${ }^{* *} P<0.001$ vs APAP group. TAC: total antioxidant capacity (A); TTGs: total thiol groups (B); GSH: glutathione (C). 

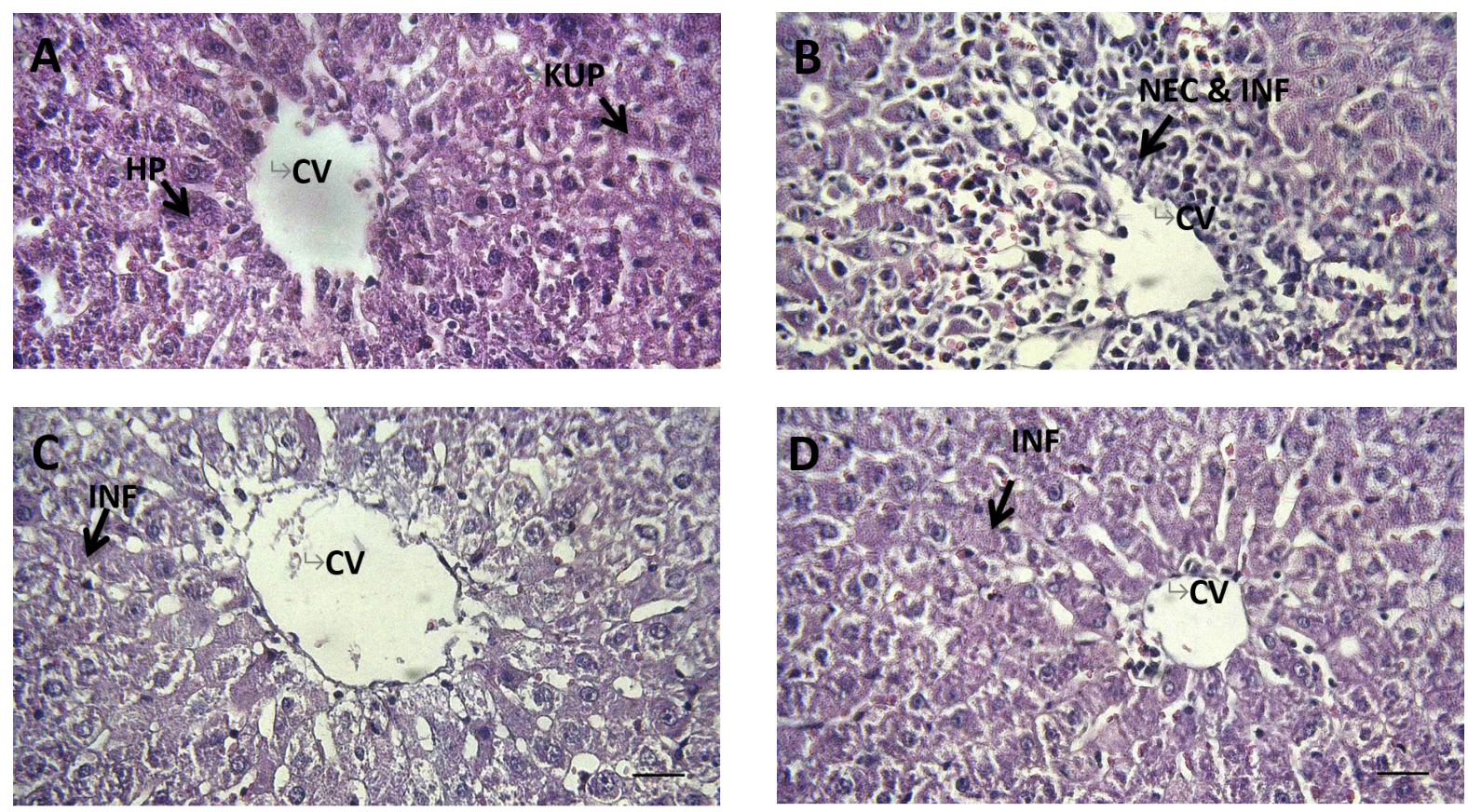

Figure 4 Acetaminophen (APAP) (2 g/kg); C, Aj extract (50 mg/kg) + APAP (2 g/kg); D, Aj extract (100 mg/kg) + APAP (2 g/kg). Original magnification of all images is $\times 40$. CV, central vein; HP, hepatocyte; KUP: Kupfer cells; NEC: necrosis; INF: infiltration.

tively) levels, were decreased in liver tissue compared to control group $(\mathrm{P}<0.001)$. In addition, a significant improvement was observed in TTGs $(\mathrm{P}<0.01)$, and GSH $(\mathrm{P}$ $<0.05)$ levels following pre-treatment with the $\mathrm{Aj}$ extract (Fig. 3B and 3C, respectively). In this regard, the levels of NO and LPO were decreased in treatment groups $(\mathrm{P}<$ 0.001).

\subsection{Histopathological experiments}

As shown in Fig. 4, mononuclear cell infiltration, dilation of sinusoids, vascular congestion, necrosis as well as the increased number of Kupffer cells were found in hepatic tissue of APAP-treated animals. Following the pretreatment with various amount of $\mathrm{Aj}$ extract, a significant improvement was observed in some of the pathological symptoms such as necrosis and vascular congestion.

\section{Discussion}

In the current study, the safe dose of the $\mathrm{Aj}$ extract was found to be $50-100 \mathrm{mg} / \mathrm{kg}$. In the high dose of $\mathrm{Aj}$ extract (200 mg/kg), a significant increase was seen in ALP and LDH serum levels alongside the histopathologic changes. It seems that Allium species contained substances that are metabolized to reactive and hepatotoxic substances when consumed high levels [23]. For instance, sodium n-propylthiosulfate and $\mathrm{N}$-propyl disulfide are very toxic organosulfur compounds present in some of the Allium species. These phytochemical constituents may cause a remarkable decrease in the activity of glucose-6-phosphate dehydrogenase (G6PD), whereas the latter can reduce glutathione level in the erythrocyte [24]. This changes leads to the vulnerability of erythrocyte to oxidative damage and subsequent hemolytic anemia, which can be associated with an increase in some serum biomarkers, especially LDH [25].

Contrary to our findings, Kalantari et al. showed that $\mathrm{Aj}$ extract had therapeutic effects at doses of 1000 and 2000 $\mathrm{mg} / \mathrm{kg}$ in hepatotoxicity induced by bromobenzene, in mice model [26]. This discrepancy could be explained due to the difference in the type of extract and animal models. As our data shown, APAP could induce liver toxicity and increase the levels of ALT, AST, ALP, and LDH which was consistent with the other literature [27-30]. When the integrity of the hepatocellular membrane disappeared, ALT and AST leaked into the bloodstream, and their level increased in blood [26]. The increased levels of ALP could be because of the biliary tract injury and/or increased biliary pressure $[31,32]$. In the groups receiving the Aj extract, a remarkable decrease was observed in liver indices which may be associated with improving the integrity of the hepatocellular membrane in animals.

Since the toxicity of APAP has been associated with oxidative pathways, LPO, and NO levels (as oxidant markers), TAC, TTGs and GSH levels (as antioxidant indices), were assessed in the hepatic tissue. Significant increase in NO and LPO levels and reduction of antioxidant indices, such as TTGs, GSH and TAC, indicate the occurrences of oxidative stress which is in line with the other literature [33-37]. Overall, NAPQI has been known as the major metabo- 
lite of APAP, which in high levels is caused reduction in the hepatocyte GSH levels. Following APAP poisoning, the excessive production of NAPQI breaks the SH-group in macromolecules, such as proteins, nucleic acids, and membranes, leading to hepatocellular dysfunction. An increased in LPO level may lead to the membrane detriment and alter the homeostasis of calcium after decreasing GSH levels and thiol storages [38].

Additionally, increased NO may be associated with APAP-inducing effects on the nitric oxide synthase (NOS) and occurrence of nitrosative stress [39]. The experimental studies showed that the excess NO reacted with superoxide anion to make peroxynitrite radicals [1, 40-42]. The role of the peroxynitrite radicals in the development of hepatic failure is confirmed through the NOS inhibitor and subsequently inhibition of endogenous NO generation as described by Gardner et al. [39]. These radicals deplete the intracellular reduced GSH leading to the increase in the vulnerability to the oxidative stress [43]. In treatment groups, a remarkable reduction was found in liver oxidant indexes (LPO and NO). Furthermore, this extract improved liver antioxidant state that may be associated with the antioxidant-related properties of Allium species $[44,45]$. It seemed that the high values of sulfur-containing constituents in the Allium species like diallyl sulfide, S-methyl cysteine 2,5-dimethylthiophene and 1-propylmercaptan could recover TTGs and GSH levels in treated rats [46]. Previously, Chiu et al. showed that 2,5-dimethylthiophene and 1-propylmercaptan may contribute to the antioxidative and antimutagenic properties of Allium species [47]. Diallyl sulfide, a garlic constituent, protects the liver from the warm hepatic ischemia-reperfusion injury through decreasing oxidative stress and inhibition of CYP2E1 [48]. S-methyl cysteine is effective in improving inflammation, and oxidative stress in rats fed with fructose rich diet [49].

Despite an increase in TTM and GSH contents there was no increase in TAC. This might be due to the fact that the FRAP cannot be useful in precisely determining the thiol-containing mixtures because this experiment does not totally react with thiol-typeantioxidants like glutathione [50]. However, the phytochemical analysis of the some of the Allium species showed the presence of several phytoconstituents such as, phenols and flavonoids which may have therapeutic effects against liver oxidative damages $[51,52]$.

\section{Conclusion}

This study showed that, although the Aj extract was toxic at the amount of $200 \mathrm{mg} / \mathrm{kg}$, its lower doses had protective effects on APAP hepatotoxicity. The Aj extract may moderate liver damage through decreasing the oxidant markers (LPO and NO) and restoring the antioxidant thiol groups that it could improve the oxidative/antioxidant balance in hepatic tissue. Our findings may expand the applications of Aj plant and offer an herbal supplement with antioxidant and hepatoprotective properties in the human diet.

\section{Acknowledgment}

The financial support for conducting the present study was provided by the Vice-chancellor of Research and Technology, Hamadan University of Medical Sciences, Hamadan, I.R. Iran (Grant No. 9605032795).

\section{Conflict of interest}

No conflicts of interest were declared by the authors.

\section{References}

1. Yoon E, Babar A, Choudhary M, Kutner M, Pyrsopoulos N, et al. Acetaminophen-induced hepatotoxicity: a comprehensive update. J Clin Transl Hepatol. 2016;4(2):131-42.

2. Abiko Y, Ishii I, Kamata S, Tsuchiya Y, Y. Watanabe, H. Ihara, et al. Formation of sulfur adducts of N-acetyl-p-benzoquinoneimine, an electrophilic metabolite of acetaminophen in vivo: participation of reactive persulfides. Chem Res Toxicol. 2015;28(9):1796-1802.

3. Walker, V, Mills GA, Anderson ME, Ingle BL, Jackson JM, Moss CL, et al. The acetaminophen metabolite $\mathrm{N}$-acetyl-p-benzoquinone imine (NAPQI) inhibits glutathione synthetase in vitro; a clue to the mechanism of 5-oxoprolinuric acidosis. Xenobiotica. 2017; 47(2):164-75.

4. Nili-Ahmadabadi A, Borzouee Z, Ahmadimoghaddam D, Firozian F, Dastan D. The occurrence of acetaminophen/ codeine as an adulterant in herbal analgesic supplements in Hamadan, Iran: A pilot study. Complement Ther Med. 2019;42: 223-5.

5. Karimi A, Majlesi M, Rafieian-Kopaei M. Herbal versus synthetic drugs; beliefs and facts. J Nephropharmacol. 2015;4(1):27-30.

6. Nili-Ahmadabadi A, Sedaghat M, Ranjbar A, Poorolajal J, Nasiripour H, Ahmadabadi M. Quantitative analysis and health risk assessment of methanol in medicinal herbal drinks marketed in Hamadan, Iran. J Appl Pharm Sci. 2016;6(7):49-52.

7. Lorigooini Z, Ayatollahi SA, Amidi S, Kobarfard F. Evaluation of anti-platelet aggregation effect of some Allium species. Iranian journal of pharmaceutical research. Iran J Pharm Res. 2015;14(4):1225-1231.

8. Suleria HAR, Butt MS, Khalid N, Sultan S, Raza A, Aleem M, et al. Garlic (Allium sativum): diet based therapy of 21st century-a review. Asian Pac J Trop Dis. 2015;5(4):271-8.

9. Praticò G, Gao Q, Manach C, Dragsted LO. Biomarkers of food intake for Allium vegetables. Genes \& nutrition. 2018;13(1):34. doi:org/10.1186/s12263-018-0624-4.

10. Rastogi S, Pandey MM, Rawat A. Traditional herbs: a remedy for cardiovascular disorders. Phytomedicine. 2016;23(11):1082-9.

11. Aslani N, Entezari MH, Askari G, Maghsoudi Z, Maracy MR. Effect of garlic and lemon juice mixture on lipid profile and some cardiovascular risk factors in people 30-60 years old with moderate hyperlipidaemia: A randomized clinical trial. Int J Prev Med. 2016;7:95. doi: 10.4103/2008- 
7802.187248 .

12. Wang HP, Yang J, Qin LQ, Yang XJ. Effect of garlic on blood pressure: A meta-analysis. J Clin Hypertens (Greenwich). 2015;17(3):223-31.

13. Zeng T, Zhang C.-L, Zhao X.-L, Xie K.-Q. The roles of garlic on the lipid parameters: a systematic review of the literature. Crit Rev Food Sci Nutr. 2013;53(3):215-30.

14. Beretta HV, Bannoud F, Insani M, Berli F, Hirschegger P, Galmarini CR, et al. Relationships between bioactive compound content and the antiplatelet and antioxidant activities of six allium vegetable species. Innov Food Sci Emerg Technol. 2017;55(2):266-75.

15. Kim S, Kim D.-B, Jin W, Park J, Yoon W, Lee Y, et al. Comparative studies of bioactive organosulphur compounds and antioxidant activities in garlic (Allium sativum L.), elephant garlic (Allium ampeloprasum L.) and onion (Allium cepa L.). Nat Prod Res. 2018;32(10):1193-7.

16. Gholami A, Arabestani MR, Ahmadi M. Evaluation of antibacterial activity of aqueous and methanol extracts of Allium Jesdianum plant on a number of pathogenic bacteria resistant to antibiotics. Pajouhan Scientific Journal. 2016;14(4):18-26.

17. Shahrokh S, Vahedi G, Khosravi A, Mahzoonieh MR, Ebrahimi A, Sharifzadeh A, et al. In vitro antifungal activity of aqueous-ethanolic extract of Allium jesdianum against fluconazole-susceptible and-resistant human vaginal Candida glabrata isolates. J Herbmed Pharmacol. 2017;6:136140.

18. Harchegani AL, Hemmati AA, Nili-Ahmadabadi A, Darabi B, Shabib S. Cromolyn sodium attenuates paraquat-induced lung injury by modulation of proinflammatory cytokines. Drug Res. 2017;67(05):283-8.

19. Nili-Ahmadabadi A, Ali-Heidar F, Ranjbar A, Mousavi L, Ahmadimoghaddam D, Larki-Harchegani A, et al. Protective effect of amlodipine on diazinon-induced changes on oxidative/antioxidant balance in rat hippocampus. Res Pharm Sci. 2018;13(4):368-76.

20. Hassani S, Maqbool F, Salek-Maghsoudi A, Rahmani S, Shadboorestan A, Nili-Ahmadabadi A, et al. Alteration of hepatocellular antioxidant gene expression pattern and biomarkers of oxidative damage in diazinon-induced acute toxicity in Wistar rat: a time-course mechanistic study. EXCLI J. 2018;17:57-71.

21. Zeinvand-Lorestani H, Nili-Ahmadabadi A, Balak F, Hasanzadeh G, Sabzevari O. Protective role of thymoquinone against paraquat-induced hepatotoxicity in mice. Pestic Biochem Physiol. 2018;148:16-21.

22. Nili-Ahmadabadi A, Alibolandi P, Ranjbar A, Mousavi L, Nili-Ahmadabadi H, Larki-Harchegani A, et al. Thymoquinone attenuates hepatotoxicity and oxidative damage caused by diazinon: an in vivo study. Res Pharm Sci. 2018;13(6):500-8.

23. Cope R. Allium species poisoning in dogs and cats. Vet Med Int. 2005;100(8):562-6.

24. Salgado BS, Monteiro LN, Rocha NS. Allium species poisoning in dogs and cats. J Venom Anim Toxins Incl Trop Dis. 2011;17(1):4-11.

25. Koseoglu M, Hur A, Atay A, Cuhadar S. Effects of hemolysis interference on routine biochemistry parameters. Biochem Medica. 2011;21(1):79-85.

26. Kalantari H, Shamsi Ehsan T, Samimi A, Kheradmand P,
Shirani M. Histopathological and Biomedical parameters Determination in the Protective Effect of Hydroalcoholic Extract of Allium Jesdianum on Hepatotoxicity Induced by Bromobenzene in Mice. Iran J Pharm Res. 2018;14(2):1524.

27. Zhang J, Song Q, Han X, Zhang Y, Zhang Y, Zhang X, et al. Multi-targeted protection of acetaminophen-induced hepatotoxicity in mice by tannic acid. Int Immunopharmacol. 2017;47:95-105.

28. Adam GO, Rahman MM, Lee S.-J, Kim G.-B, Kang H.-S, Kim J.-S, et al. Hepatoprotective effects of Nigella sativa seed extract against acetaminophen-induced oxidative stress. Asian Pac J Trop Biomed. 2016;9(3):221-7.

29. Alam J, Mujahid M, Jahan Y, Bagga P, Rahman MA. Hepatoprotective potential of ethanolic extract of Aquilaria agallocha leaves against paracetamol induced hepatotoxicity in SD rats. J Tradit Complement Med. 2017;7(1):9-13.

30. K Chellappan D, Ganasen S, Batumalai S, Candasamy M, Krishnappa P, Dua K, et al. The protective action of the aqueous extract of Auricularia polytricha in paracetamol induced hepatotoxicity in rats. Recent Pat Drug Deliv Formul. 2016;10(1):72-6.

31. Yan J.-Y, Ai G, Zhang X.-J, Xu H.-J, Huang Z.-M. Investigations of the total flavonoids extracted from flowers of Abelmoschus manihot (L.) Medic against a-naphthylisothiocyanate-induced cholestatic liver injury in rats. J Ethnopharmacol. 2015;172: 202-13.

32. Bhakuni GS, Bedi O, Bariwal J, Kumar P. Hepatoprotective activity of morin and its semi-synthetic derivatives against alcohol induced hepatotoxicity in rats. Indian J Physiol Pharmacol. 2017;61(2):175-90.

33. Noh J.-R, Kim Y.-H, Hwang J. H, Choi D.-H, Kim K.-S, Oh W.-K, et al. Sulforaphane protects against acetaminophen-induced hepatotoxicity. Food Chem Toxicol. 2015;80:193-200.

34. Honmore V, Kandhare A, Zanwar AA, Rojatkar S, Bodhankar S, Natu A. Artemisia pallens alleviates acetaminophen induced toxicity via modulation of endogenous biomarkers. Pharm Biol. 2015;53(4):571-81.

35. Wang X, Wu Q, Liu A, Anadón A, Rodriguez J.-L, Martinez-Larranaga M.-R, et al. Paracetamol: overdose-induced oxidative stress toxicity, metabolism, and protective effects of various compounds in vivo and in vitro. Drug Metab Rev. 2017;49(4):395-437.

36. Kandemir F, Kucukler S, Eldutar E, Caglayan C, Gülçin I. Chrysin protects rat kidney from paracetamol-induced oxidative stress, inflammation, apoptosis, and autophagy: A multi-biomarker approach. Sci Pharm. 2017;85(1):4. doi: 10.3390/scipharm85010004.

37. Amin KA, Hashem KS, Alshehri FS, Awad ST, Hassan MS. Antioxidant and hepatoprotective efficiency of selenium nanoparticles against acetaminophen-induced hepatic damage. Biol Trace Elem Res. 2017;175(1):136-145.

38. Nchouwet ML, Wansi N, Kodjio N, Poualeu K, Nkeng-Efouet A, Kamanyi A. Hepatoprotective and antioxidant effect of stem barks extracts: methanolic and aqueous of Pseudocedrela kotschyi (Meliaceae) on paracetamol-induced hepatic damage in rats. Asian J Biomed Pharm Sci. 2017;7:1-9.

39. Gardner CR, Heck DE, Yang CS, Thomas PE, Zhang XJ, DeGeorge GL, Laskin JD, Laskin DL. Role of nitric oxide in acetaminophen-induced hepatotoxicity in the rat. Hepa- 
tology. 1998;27(3):748-54.

40. Ramachandran A, Jaeschke H. Acetaminophen toxicity: novel insights into mechanisms and future perspectives. Gene Expr. 2018;18(1):19-30.

41. Chi D, Rong L, Wang SE, Wang X, Wang X, Ma L, et al. Role of oxidative/nitrative stress in hepatic encephalopathy induced by thioacetamide. Pharmazie. 2011; 66(5): 378-81.

42. Du K, Farhood A, Jaeschke H. Mitochondria-targeted antioxidant Mito-Tempo protects against acetaminophen hepatotoxicity. Arch Toxicol. 2017;91(2):761-73.

43. Shi C, Zhou X, Zhang J, Wang J, Xie H, Wu Z. $\alpha$-Lipoic acid protects against the cytotoxicity and oxidative stress induced by cadmium in HepG2 cells through regeneration of glutathione by glutathione reductase via Nrf2/ARE signaling pathway. Environ Toxicol Pharmacol. 2016;45:274-81.

44. Fratianni F, Ombra MN, Cozzolino A, Riccardi R, Spigno P, Tremonte $\mathrm{P}$, et al. Phenolic constituents, antioxidant, antimicrobial and anti-proliferative activities of different endemic Italian varieties of garlic (Allium sativum L.). J Funct Foods. 2016;21:240-8.

45. Martins N, Petropoulos S, Ferreira IC. Chemical composition and bioactive compounds of garlic (Allium sativum L.) as affected by pre-and post-harvest conditions: A review. Food Chem. 2016;211:41-50.

46. Zeng Y, Li Y, Yang J, Pu X, Du J, Yang X, et al. Therapeutic role of functional components in alliums for preventive chronic disease in human being. Evid Based Complement Alternat Med. 2017. doi: 10.1155/2017/9402849.

47. Chiu CK, Chen TY, Lin JH, Wang CY, Wang BS. Protective effects of five allium derived organosulfur compounds against mutation and oxidation. Food Chem. 2016;197:82935.

48. Shaik IH, George JM, Thekkumkara TJ, Mehvar R. Protective effects of diallyl sulfide, a garlic constituent, on the warm hepatic ischemia-reperfusion injury in a rat model. Pharm Res. 2008;25(10):2231-42.

49. Thomas S, Senthilkumar GP, Sivaraman K, Bobby Z, Paneerselvam S, Harichandrakumar KT. Effect of s-methylL-cysteine on oxidative stress, inflammation and insulin resistance in male Wistar rats fed with high fructose diet. Iran J Med Sci. 2015;40(1):45-50.

50. Çekiç SD, Başkan KS, Tütem E, Apak R. Modified cupric reducing antioxidant capacity (CUPRAC) assay for measuring the antioxidant capacities of thiol-containing proteins in admixture with polyphenols. Talanta. 2009;79(2):344-51.

51. Rahimi-Madiseh M, Heidarian E, Kheiri S, Rafieian-Kopaei M. Effect of hydroalcoholic Allium ampeloprasum extract on oxidative stress, diabetes mellitus and dyslipidemia in alloxan-induced diabetic rats. Biomed Pharmacother. 2017;86:363-7.

52. Naji KM, Al-Shaibani ES, Alhadi FA, D'souza MR. Hepatoprotective and antioxidant effects of single clove garlic against CCl4-induced hepatic damage in rabbits. BMC Complement Altern Med. 2017;17(1):411. doi: 10.1186/ s12906-017-1916-8. 